\title{
tic\&société
}

Vol. 2, $n^{\circ} 1 \mid 2008$

Tic et santé

\section{Les réseaux de santé ou la rencontre de la santé et des TIC pour décloisonner le système de santé} français

\section{Christian Bourret}

\section{(2) OpenEdition \\ Journals \\ Édition électronique \\ URL : http://journals.openedition.org/ticetsociete/396 \\ DOI : 10.4000/ticetsociete.396}

\section{Éditeur}

Association ARTIC

\section{Référence électronique}

Christian Bourret, « Les réseaux de santé ou la rencontre de la santé et des TIC pour décloisonner le système de santé français », tic\&société [En ligne], Vol. 2, n 1 | 2008, mis en ligne le 13 octobre 2008 , consulté le 19 avril 2019. URL : http://journals.openedition.org/ticetsociete/396 ; DOI : 10.4000/

ticetsociete.396 


\title{
Les réseaux de santé ou la rencontre de la santé et des TIC pour décloisonner le système de santé français
}

\author{
Christian BOURRET \\ Maître de conférences en sciences de l'information et de la communication. \\ Université de Paris Est \\ IFIS \\ Cité Descartes \\ 5 boulevard Descartes \\ Champs sur Marne \\ 77454 Marne-la-Vallée Cedex 2 \\ France \\ bourret@univ-mlv.fr
}

Christian Bourret est maître de conférences en sciences de l'information et de la communication (SIC) à l'Université de Paris-Est (Marne-la-Vallée). Diplômé de l'École des Hautes Études Commerciales (HEC), docteur, il est directeur de l'Institut Francilien d'Ingénierie des Services (IFIS). Ses travaux de recherche portent sur l'analyse des usages de l'information et des processus communicationnels dans les organisations d'interface de la protection sociale (en particulier réseaux de santé). Centrés sur la construction de connaissances collectives, notamment dans une perspective d'évaluation-amélioration, ils relèvent d'une approche d'intelligence territoriale de proximité (construction de territoires de pratiques). II est membre du programme de recherche en intelligence économique (CNRS). 


\title{
Les réseaux de santé ou la rencontre de la santé et des TIC pour décloisonner le système de santé français
}

\begin{abstract}
Résumé
En France, les réseaux de santé constituent depuis le milieu des années 1980 une tentative de réponse originale aux cloisonnements du système de santé. Les enjeux de maîtrise de l'information et d'amélioration des processus de communication sont essentiels dans l'affirmation de ces organisations d'interface entre la médecine de ville et l'hôpital. Leur émergence correspond à une approche globale de la santé (complexité). Espaces innovants de recomposition des positionnements, ils se construisent progressivement par les interactions entre acteurs, à la fois humains et dispositifs techniques, autour de nouvelles pratiques coopératives. L'amélioration de la coordination entre tous les acteurs est déterminante. Après une phase initiale correspondant à la constitution de réseaux d'acteurs sociaux, leur rencontre avec de nouveaux outils relevant des TIC : systèmes d'information et dossiers médicaux informatisés, ouvre de nouvelles perspectives. Leur avenir est néanmoins loin d'être assuré. Le succès de ces espaces de négociation repose sur la construction de la confiance entre tous les acteurs avec une territorialisation de nouvelles pratiques de santé. Leurs enjeux sont représentatifs de ceux de toute notre société.
\end{abstract}

Mots-clés : réseaux, cloisonnements, santé, coordination, complexité.

\begin{abstract}
In France, since the middle of the 1980s health networks have proved to be an original attempt to transcend the compartmentalization of the Health System. The challenges of controlling information and improving communication processes are essential in positioning these organizations at the interface between primary care and the hospital. Their emergence corresponds to a global approach to health (complexity). As innovative spaces of positional reorganization, they are built gradually thanks to interactions between both human actors and technical devices, through new
\end{abstract}




\section{Christian BOURRET}

cooperative practices. Improved coordination between all the actors is determinant. After an initial phase dealing with the constitution of social actors' networks, their meeting with new ICT tools: Information Systems and EHR (Electronic Health Records) opens new prospects. Nevertheless their future is far from guaranteed. The success of these negotiation spaces rests on building trust between all the actors and the territorialization of new health practices of health. Their stakes are representative of those of our society as a whole.

Keywords: networks, compartmentalization, healthcare, coordination, complexity.

\section{Resumen}

En Francia, las redes de salud constituyen, desde mediados de los años ochenta, una tentativa de respuesta original a las divisiones del sistema de salud. La gestión de la información y la mejora de los procesos de comunicación éstán en la base de estas organizaciones de interfaz entre la medicina de atención primaria y el hospital. Su aparición corresponde a un enfoque global de la salud (complejidad). Las interacciones entre los protagonistas -humanos y técnicos- en torno a nuevas prácticas cooperativas suponen espacios innovadores que recomponen las posiciones.

La mejora de la coordinación entre todos los agentes es determinante. Después de una fase inicial que corresponde a la constitución de redes de agentes sociales, su encuentro con nuevas herramientas relacionadas las TIC -sistemas de información e historiales clínicos informatizados- abren nuevas perspectivas. Su futuro es sin embargo, incierto. El éxito de estos espacios de negociación se basa en la construcción de la confianza entre todos aquellos agentes que intervienen y en la territorialización de nuevas prácticas de salud. Los retos son los de nuestra sociedad entera.

Palabras clave: redes, divisiones, salud, coordinación, complejidad. 
Les réseaux de santé ou la rencontre de la santé et des TIC pour décloisonner le système de santé français

\section{Introduction}

Dans tous les pays industrialisés, à des degrés divers, les systèmes de santé sont en crise. La question des cloisonnements (séparations très marquées entre les activités d'organisations ou d'acteurs individuels qui devraient davantage coopérer) et des coûts qui en découlent est devenue centrale, notamment en France. L'État et l'Assurance Maladie ont cherché des solutions dans une meilleure maîtrise de l'information et une amélioration des processus de communication. Une réponse a consisté en l'affirmation de nouvelles organisations d'interface entre la médecine de ville et l'hôpital : les réseaux de santé.

Cet article s'appuie sur des coopérations avec des réseaux de santé et des organismes de protection sociale, nées autour d'une option de master (Ingénierie des réseaux de santé): études de cas construits à partir d'observations sur le terrain, entretiens avec des acteurs des réseaux de santé, participations à des séminaires ou colloques, implication dans l'évaluationamélioration de réseaux de santé.

Nous montrerons tout d'abord le besoin d'organisations d'intermédiation pour décloisonner le système de santé. Nous étudierons ensuite les conditions d'émergence des réseaux de santé, comme organisations construites par les interactions entre acteurs autour de nouvelles pratiques coopératives. Nous analyserons la rencontre des réseaux de santé avec de nouveaux outils relevant des TIC (systèmes d'information, dossiers médicaux personnels, etc.) pour décloisonner en favorisant les coopérations, l'implication des patients et une nouvelle territorialisation des pratiques de santé. Nous terminerons en nous interrogeant sur l'avenir des réseaux de santé.

\section{Un impératif : décloisonner les systèmes de santé}

\subsection{La prise de conscience de l'importance des cloisonnements}

Le défi des systèmes de santé est partout le même : «to provide ready access at reasonable cost » (Fuchs, 1998, p. 75). Selon la Haute Autorité de Santé, les cloisonnements sont des facteurs majeurs de coûts et de non productivité. Ils sont nombreux en France. Au niveau des organisations, il y a d'abord ce clivage entre l'État (ministère de la Santé) et l'Assurance Maladie, qui n'a pas été modifié par la réforme d'août 2004, celui entre la médecine de ville (privée) et l'hospitalisation à la fois publique avec les hôpitaux, et privée avec les cliniques, et enfin celui entre médecins et professions non médicales. 


\section{Christian BOURRET}

Dans tous les pays, on retrouve des barrières entre le traitement (cure), le soin et la santé (care) liée au social. Selon la définition de l'OMS, « la santé est un état de complet bien-être physique, mental et social, et ne consiste pas seulement en une absence de maladie ou d'infirmité ». Elle ne saurait se limiter aux soins. Cette approche globale de la santé est au cœur de la distinction entre réseaux de soins coordonnés et réseaux de santé, sur laquelle nous reviendrons. Elle suppose de faire converger différentes approches et représentations professionnelles (hôpital, ville, médecins, infirmières, travailleurs sociaux, etc.) pour construire de nouvelles pratiques impliquant un patient devenu acteur de sa santé.

\subsection{Culpabilisation, maîtrise de l'information et amélioration des processus de communication}

En analysant l'évolution en France et au Québec, D. Carré et J.-G. Lacroix ont mis en évidence « l'idéologie de la culpabilisation pour légitimer l'imposition de la solution » : le virage ambulatoire pour diminuer le poids de l'hôpital (45 \% des dépenses en France), puis le «syndrome du tout informatique », «l'informatique devant tout solutionner » (Carré et Lacroix dir., 2001, p. 41 et p. 9).

D'après Grimson et al., «The present inability to share information across systems and between care organisations represents one of the major impediments to progress toward shared care and cost containment » (Grimson et al., 2000, p. 49). Dès 1998, le Royaume-Uni a considéré la maîtrise de l'information comme un enjeu essentiel (NHS Executive, 1998). Au Canada, le rapport Romanow (2002) a fait une large place aux usages de l'information. Aux États-Unis, le Committee on Government Reform a considéré, en 2005, l'introduction des technologies de l'information dans la santé comme la « dernière frontière » (Committee on Government Reform, 2005).

En France, les lois d'août 2004 ont également insisté sur ces enjeux, en reprenant les préconisations du rapport Fieschi (2003) au titre significatif : « Les données du patient partagées: la culture du partage et de la qualité des informations pour améliorer la qualité des soins ", mais aussi pour développer l'efficience de la gestion du système. Ce développement des usages de l'information relève du «procès d'informationnalisation » analysé par B. Miège (2004, p. 41). 
Les réseaux de santé ou la rencontre de la santé et des TIC pour décloisonner le système de santé français

\section{Une réponse aux cloisonnements en France : les réseaux de santé}

\subsection{Une approche globale d'“ intelligence de la complexité »}

Les réseaux sont à la mode. M. Castells a qualifié notre société de "société en réseaux » (1998). Selon H. Sérieyx et al. (1996), ils constituent une manière d'aborder la complexité de notre monde. Alors que la pyramide divise le travail, le réseau multiplie la valeur ajoutée des contributions, la pyramide garantit l'ordre, le réseau assure la vie. Pour d'autres, plus critiques comme P. Musso (2003), ils sont devenus une véritable idéologie et un mot passe-partout favorisant toutes les confusions.

Dès son origine (Grèce antique), la notion de réseau, associée à celle de maillage, est ambivalente : le réseau relie (tissage) ou sépare et capture (filet de pêche, de chasse ou de combat cf. le gladiateur rétiaire à Rome). Cette ambivalence des réseaux justifie une approche «dialogique " reposant sur l'analyse des tensions entre des doubles logiques: à la fois opposition et complémentarité relevant d'une approche globale d'«intelligence de la complexité » (Morin et Le Moigne, 2003).

V.R. Fuchs a mis en évidence différentes tensions au sein des systèmes de santé. Tout d'abord de l'individuel et du collectif, ou de la «jungle» et du « zoo ». Trop de liberté laissée aux individus amène l'insécurité pour tous de la loi de la jungle. En revanche, le poids de l'État ou de toute forme d'organisation collective peut déboucher sur la sécurité du zoo, obtenue aux dépens de la liberté (Fuchs, 1998, p. 26). II rejette ensuite point de vue «romantique » et point de vue "monotechnique » sur la santé. L'approche "romantique », correspondant à la formule : "la santé n'a pas de prix ", nie, en période de ressources limitées, l'obligation d'effectuer des choix. En revanche, le point de vue «monotechnique ", souvent celui des ingénieurs ou des médecins, oublie la diversité des préférences individuelles (Fuchs, 1998, p. 5) avec le danger d'une approche «technocentrée ».

Une autre approche "dialogique » est celle de l'ordre et du désordre, centrale dans des organisations innovantes comme les réseaux de santé. Cette "dialogie " de l'innovation et du contrôle se retrouve dans l'analyse de S. Grosjean et L. Bonneville sur les "logiques d'implantation des TIC dans le secteur de la santé » (2007) avec la tension entre une logique technicoéconomique d'impératif productif et une logique médico-intégrative d'impératif créatif. C'est aussi l'opposition entre logique du prescrit par le haut (État, Assurance Maladie) et du construit sur le terrain. En favorisant le partage puis la 
production collective d'informations, les TIC peuvent aider à dépasser ces oppositions en aidant à la convergence des représentations.

\subsection{Le besoin d'organisations d'interface et la rencontre avec la notion de projet}

Le besoin de nouvelles organisations d'interface est très fort en France où existent une logique de corps (Rosanvallon, 1998) et un individualisme marqué, notamment chez les médecins de ville : le patient paie à l'acte le médecin puis se fait rembourser par les caisses de sécurité sociale ou les mutuelles. La responsabilité médicale est individuelle. Pour défendre la profession médicale, un ordre des médecins a été créé en 1942. II a fallu attendre le printemps 2007 pour voir la création d'un ordre des infirmiers.

L'approche par les réseaux rencontre une autre notion clé de notre société post-industrielle : le culte du projet (Boutinet, 2006). Le projet essaie de concilier l'anticipation (futur) avec la réalisation en s'appuyant sur l'expérience (mémoire du passé). Créant un nouvel espace de coopération entre la médecine de ville et l'hôpital, les réseaux de santé se sont développés depuis le milieu des années 1980 de deux manières différentes: réseaux de santé et réseaux de soins. Correspondant à une approche globale du patient, les réseaux de santé sont apparus dans des zones difficiles (banlieues défavorisées) et pour des populations en situation souvent précaire, en particulier pour répondre au développement du SIDA. La seconde approche est davantage celle d'organismes de sécurité sociale, d'assureurs ou d'hôpitaux qui, en s'inspirant des HMOs (Health Maintenance Organizations) américaines, ont essayé de mieux coordonner les soins pour maîtriser les dépenses (réseaux coordonnés de soins).

Les réseaux de santé ont été reconnus par les ordonnances d'avril 1996 en tant qu'expérimentations à vocation surtout tarifaire (réseaux de soins) puis consacrés par la loi du 4 mars 2002 sur les Droits des malades et la qualité du système de santé qui en propose une définition synthétique : "Les réseaux de santé ont pour objet de favoriser l'accès aux soins, la coordination, la continuité ou l'interdisciplinarité des prises en charge sanitaires, notamment de celles qui sont spécifiques à certaines populations, pathologies ou activités sanitaires. Ils assurent une prise en charge adaptée aux besoins de la personne tant sur le plan de l'éducation à la santé, de la prévention, du diagnostic, que des soins. Ils peuvent participer à des actions de santé publique. Ils procèdent à des actions d'évaluation afin de garantir la qualité de leurs services et prestations ».

Une brochure de juin 2006 de la Haute Autorité de Santé (HAS) précise cette définition, présentant les réseaux de santé comme "une démarche d'amélioration de la qualité » (Haute Autorité de Santé, 2006). Elle insiste sur 
Les réseaux de santé ou la rencontre de la santé et des TIC pour décloisonner le système de santé français

l'évaluation et l'amélioration des pratiques autour d'un programme de soins adaptés à chaque patient (protocole). Chaque réseau est différent. Une autre définition d'un réseau de santé peut être l'organisation de la coopération de professionnels de santé qui se construit à chaque fois autour du patient et de sa famille.

\section{Le levier de la rencontre avec les TIC pour améliorer la coordination}

\subsection{Une construction progressive}

Les réseaux de santé se sont affirmés progressivement. Selon B. El Ghozi (Créteil), "dix années ont été nécessaires pour créer une culture commune » (2005, p. 20). Pour lui, les réseaux de santé se sont développés en trois étapes : celle de l'émergence à partir du terrain, puis celle de la rencontre et de l'adaptation des réseaux de santé aux logiques des institutions qui financent (notamment l'évaluation) et, à présent, celle de la construction du partenariat avec l'usager (El Ghozi, 2005, p. 20).

Les patients s'impliquent davantage, notamment à travers de puissantes associations fédérées dans le CISS ou Collectif Interassociatif Sur la Santé. L'affirmation du rôle des patients devenus acteurs de leur santé est allée de pair avec le développement des usages des TIC. Le recours aux technologies Internet a modifié au profit du patient la traditionnelle asymétrie d'information entre le médecin et le patient. L. Sfez a situé cette évolution dans le cadre de l' « utopie de la santé parfaite » (Sfez dir., 2001). Mieux informés, les patients sont devenus plus revendicatifs. Ils ne se contentent plus d'une logique de moyens mais exigent désormais des résultats, et, en cas d'échec, n'hésitent pas à aller devant les tribunaux.

\subsection{De nouveaux outils pour des pratiques coopératives coordonnées}

Les différences entre réseaux de santé et réseaux de soins peuvent correspondre à des usages différents des TIC. Les réseaux de santé se sont surtout développés à partir de réseaux relationnels de professionnels de santé. Les dossiers médicaux des patients de ces réseaux de santé sont souvent sous forme papier. Ces réseaux reposent sur une conception du soin, un effet de réputation, des liens amicaux ou de confiance. "D'une certaine façon, un réseau préexiste au réseau ", ce qui explique "toute la difficulté qu'il y a à dépasser le noyau initial » (Schweyer, 2004, p. 42). En revanche, les réseaux de soins n'ont pas toujours échappé à l'obsession techniciste. L'opposition réseaux de santé / réseaux de soins rejoint finalement celle relevée par $\mathrm{S}$. 


\section{Christian BOURRET}

Grosjean et L. Bonneville dans les logiques d'implantation des TIC dans le secteur de la santé (2007), les réseaux de santé relevant davantage d'une logique médico-sociale intégrative, les réseaux de soins d'une logique technicoéconomique.

Pour M. Gadreau (2001), les réseaux de santé constituent avant tout une « forme de coordination émergente dans le système de santé ». L'amélioration de la coordination constitue leur raison d'être. En France, le DMP (dossier médical personnel) a été présenté par les pouvoirs publics comme l'élément central de la réforme d'août 2004. II est censé assurer à la fois la traçabilité des soins et améliorer leur qualité, avec l'objectif d'en réduire les coûts. Le projet demeure une priorité pour les pouvoirs publics, mais sa mise en place a été différée et devrait, selon eux, prendre une dizaine d'années.

Le choix du législateur français a été celui d'un dossier médical « personnel » et non pas «partagé ». II appartient au patient qui en définit les conditions d'accès et qui bénéficie d'un « droit au masquage » de certaines données. Il sera distinct des dossiers professionnels des médecins et ses données seront gérées par des « hébergeurs » privés. Dossier du patient, le DMP doit néanmoins être un dossier partagé et voir converger des données ou au moins des liens vers d'autres dossiers (dossiers de patients des réseaux de santé, dossiers hospitaliers, hospitalisation à domicile, dossier pharmacien, dossier communicant cancérologie, etc.) et vers les dossiers des médecins (cabinets médicaux), ce qui suppose d'assurer l'interopérabilité entre ces différents dispositifs-acteurs. Le DMP n'est néanmoins qu'un élément de systèmes d'information en santé que le rapport Fieschi (2003) proposait d'envisager au niveau régional. II soulignait la difficulté de les mettre en place, les mentalités françaises étant peu portées au partage des données. 


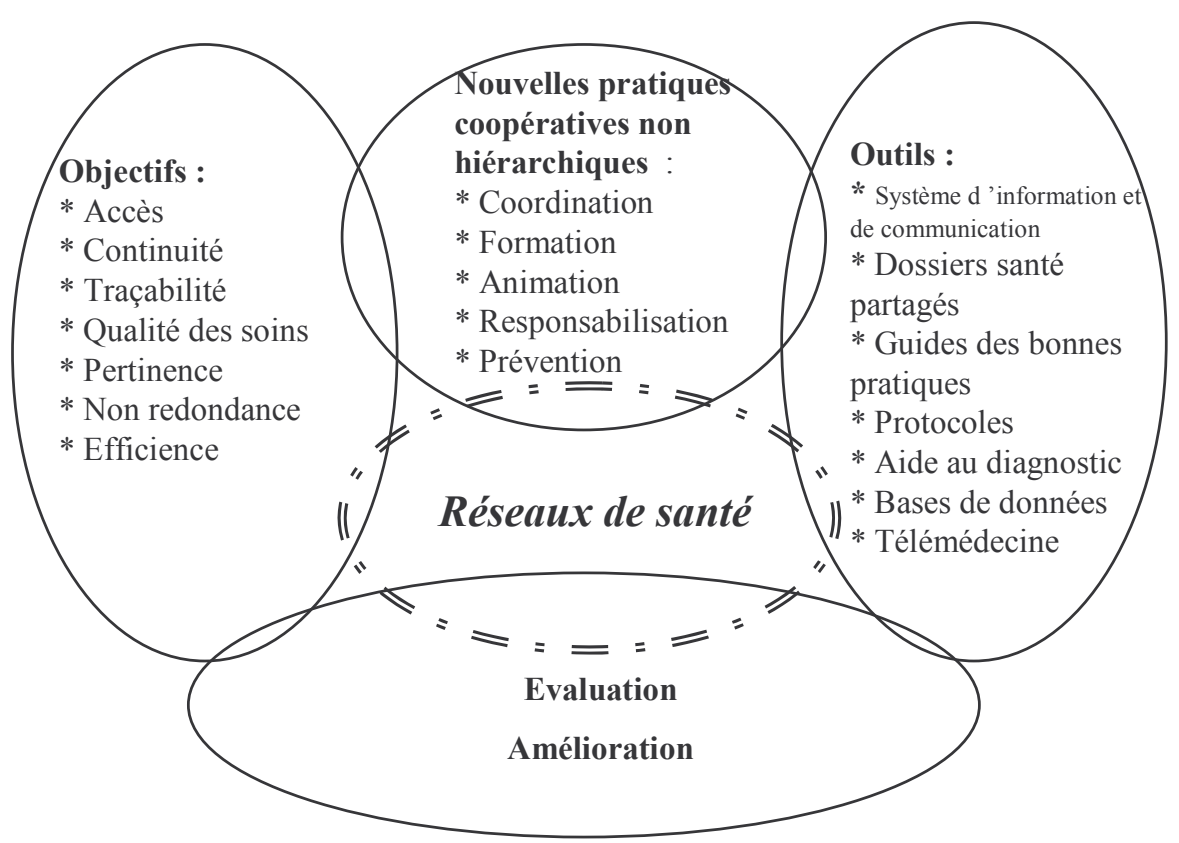

Réseaux de santé, nouvelles pratiques et outils TIC

L'informatisation du système de santé (acteurs institutionnels, opérateurs, sociétés de services spécialisées en solutions informatiques ou en hébergement de données, etc.), les nouvelles modalités de financement des établissements hospitaliers (PMSI ou Programme de Médicalisation des Systèmes d'Information et T2A ou Tarification à l'Activité), et les nouvelles formes d'exercice à distance (télémédecine), produisent de nouvelles informations qui ont aussi leur influence sur l'évolution des réseaux de santé.

Si une meilleure utilisation de l'information, allant vers son partage puis sa production collective, doit permettre d'améliorer les systèmes de santé en dépassant leurs cloisonnements, il doit y avoir des "passeurs ", acteurs à la fois individuels (micro) et en termes d'organisations (méso ou macro), permettant d'assurer ces intermédiations ou franchissements des barrières, reposant largement sur de nouvelles compétences informationnelles et 


\section{Christian BOURRET}

communicationnelles. C'est la raison d'être des réseaux de santé, en construisant de nouvelles pratiques coopératives autour de nouveaux outils.

\subsection{Dynamiques informationnelles et amélioration des processus communicationnels}

La construction progressive des réseaux de santé peut être analysée comme une synergie entre information et communication. C'est à travers le passage d'une information conçue souvent au départ par les médecins pour un usage personnel à un usage partagé que se construit progressivement autour de nouvelles pratiques coopératives l'innovation organisationnelle que constitue le réseau de santé. Ce passage d'une production individuelle à une utilisation partagée, puis à une production collective, constitue finalement une « communication généralisée processuelle », pour reprendre une formule d'A. Mucchielli, en référence au « collège invisible » de Palo Alto (Mucchielli et Noy, 2005, p. 136). Les réseaux de santé peuvent aussi être envisagés comme un « slack informationnel et communicationnel », notion proposée par A. Mayère et B. Vacher (2005). Elles partent d'une analyse de J.G. March (1991), pour qui le "slack» constitue un surplus organisationnel, une marge de manœuvre. Les contraintes de productivité le réduisent partout, voire le font disparaître. La réponse à cette diminution du «slack» dans ses formes antérieures peut résider en un «slack informationnel et communicationnel » pour rendre les acteurs non plus seulement individuellement, mais collectivement efficaces, capables de gérer les aléas, d'interpréter des situations nouvelles.

Les réseaux de santé reposent sur l'affirmation de nouvelles compétences informationnelles et communicationnelles. Ils font évoluer les frontières entre les métiers existants (médecins, infirmières, travailleurs sociaux, etc.) et apparaître de nouvelles fonctions, voire de nouveaux métiers : coordonnateurs de réseaux de santé (le plus spécifique, la coordination étant la priorité existentielle du réseau de santé), évaluateurs, gestionnaires de données pour les « hébergeurs » (Bourret, 2004), gestionnaires de cas pour améliorer le suivi des patients devenant co-acteurs de la construction de leur parcours de santé.

Les risques de dérives sont à la mesure des enjeux. Les réseaux et les TIC peuvent permettre d'améliorer la gestion du système de santé et les relations avec les patients, mais ils peuvent aussi favoriser la généralisation d'une société du contrôle (Musso, 2003), certains considérant le DMP comme un «casier sanitaire ». Le débat n'est pas nouveau. J. Ellul a souligné « l'ambivalence du progrès technique » (1990). Des systèmes d'information centralisés ont pu mettre en péril des réseaux de santé qui ont dû renoncer à leur utilisation. Les TIC peuvent aussi contribuer à aggraver les cloisonnements. Le rapport de la Cour des Comptes de 2007 sur la Sécurité Sociale a stigmatisé la multitude 
Les réseaux de santé ou la rencontre de la santé et des TIC pour décloisonner le système de santé français

d'intervenants institutionnels et a insisté sur les enjeux de l'interopérabilité (Cour des Comptes, 2007, p. 308).

II y a débat entre ceux qui sont persuadés du caractère déterministe de la technologie sur l'évolution des organisations et ceux qui insistent sur la neutralité des techniques et sur la "malléabilité organisationnelle des TIC » (Peaucelle, 2007). L'affirmation des réseaux de santé peut être appréhendée dans le cadre de la théorie de la «technologie démocratique » d'A. Feenberg. II reprend l'approche de l' «innovation concrétisante » de G. Simondon, pour réinscrire le processus de fonctionnalisation de l'objet dans un cadre contextuel plus large, rassemblant des groupes sociaux autour d'artefacts ou de systèmes d'artefacts. Pour lui, les techniques prennent des significations différentes dans des contextes sociaux différents (Feenberg, 2004, p. 210).

\section{Une organisation construite par la dynamique des interactions entre acteurs}

\subsection{Faire converger les représentations d'une grande diversité d'acteurs}

Chacun des acteurs institutionnels et humains des réseaux de santé dispose désormais de systèmes d'informations (systèmes d'informations hospitaliers, logiciels de gestion de cabinet médical, etc.) y compris le patient lui-même et ses nombreux dossiers informatisés entrant ou non dans le DMP.

Organisations-projets, les réseaux de santé peuvent être appréhendés dans le cadre d'une approche systémique relevant des logiques d'action (Bernoux, 2002, p. 47). Chaque acteur a sa propre stratégie. Dans L'acteur et le système (1977), M. Crozier et E. Friedberg ont analysé la rencontre des systèmes organisés et de la stratégie des acteurs. Dans Le pouvoir et la règle (1993), E. Friedberg a prolongé l'analyse de la «dynamique de l'action organisée ». Dans sa théorie de la structuration, A. Giddens (1987) a souligné que les propriétés structurelles des systèmes sociaux sont à la fois des conditions et des résultats des activités des agents. Les réseaux de santé constituent des espaces-projets ou formes-projets, dont les contours évoluent au rythme des interactions entre acteurs y compris dispositifs techniques.

La «théorie de la traduction " (Akrich, Callon et Latour, 2006) constitue une autre approche possible. Elle débouche sur la construction d'un "modèle de traduction ", impliquant tous les acteurs dans un langage que chacun peut comprendre et s'approprier. Selon P. Bernoux, le dynamisme des organisations dépend ainsi de leur faculté de «traduction» (2002, p. 46). Les coordonnateurs 


\section{Christian BOURRET}

des réseaux sont ainsi des «traducteurs » privilégiés. La « sociologie de l'acteur réseau " ou SAR, considérant les dispositifs techniques comme des « actants » à part entière, est intéressante pour analyser les réseaux de santé : le réseau pouvant être considéré comme un « centre de traduction » (Callon, 2006).

Les réseaux de santé se développent quand les représentations personnelles et les stratégies individuelles des acteurs se transforment progressivement en pratiques coopératives collectives. Les conditions générales de succès des réseaux ont été mises en évidence par $G$. Le Boterf. La coopération se construit par la convergence du vouloir coopérer (valorisation des contributions, confiance, etc.), savoir coopérer (apprentissage et capitalisation progressive, représentations partagées, conception d'outils communs, maîtrise des TIC) et pouvoir coopérer (instances de régulation, méthodes, équipements notamment TIC, etc.) (Le Boterf, 2004, p. 69).

Selon F.-X. Schweyer, «les réseaux qui «fonctionnent» ne font que systématiser des liens qui existent déjà, ils ne les créent pas » (Schweyer, 2005). "Le réseau rend visible une communauté professionnelle qui existe déjà [...] Ce ne sont pas les procédures ou les outils qui structurent les réseaux mais une certaine conception du soin » (Schweyer, 2005, pp. 90-92). Les réseaux de santé sont des réseaux d'acteurs sociaux avant de devenir des réseaux sociotechniques et se construisent progressivement par les interactions entre acteurs humains et artefacts techniques. La question des représentations mutuelles et de leur convergence est donc centrale (CNR, 2005, p. 31 et 71).

Pour analyser la rencontre des réseaux de santé et des TIC, il faut prendre en compte trois temporalités différentes: celle de l'apparition de nouvelles technologies (très rapide), celle de leur appropriation socioculturelle et de la construction progressive des usages, plus longue (de l'ordre d'une dizaine d'années), et celle, encore plus longue, de l'évolution des représentations sociales (plusieurs dizaines d'années). La construction de logiques sociales, correspondant à l'appropriation des outils par les différents acteurs des réseaux de santé est une des conditions majeures de leur succès (Miège, 2004)

\subsection{La négociation et la construction de la confiance}

Si la maîtrise de l'information est essentielle, les réseaux de santé constituent avant tout de nouveaux espaces communicationnels avec l'importance de la négociation «constitutive et instituante » (Grosjean et al., 2004). Pour R. Bercot, «la pérennité des réseaux dépend de la capacité de négocier des acteurs qui en composent le noyau central : négocier des moyens, un territoire, une place et une renommée » (2006, p. 35). 
Les réseaux de santé ou la rencontre de la santé et des TIC pour décloisonner le système de santé français

Selon A. Giddens, la notion de confiance est fondamentale dans les institutions de la modernité $(1994$, p. 34 et 41). S. Haber rappelle que pour N. Luhmann, la confiance est un réducteur de complexité dans la vie sociale et la condition de toute dynamique d'enrichissement des relations interhumaines (Haber, 2006, p. 51). B. Nooteboom envisage la construction de la confiance comme un processus d'apprentissage. II insiste sur le rôle d'intermédiaires dans la création ou le maintien de la confiance en réduisant le risque : "gardiens de la confiance " ou "communautés intermédiaires " (Nooteboom, 2006, p. 64 et 72). C'est bien là l'objectif majeur des réseaux de santé : décloisonner en créant de nouveaux espaces de coopérations.

F.-X. Schweyer a montré que l'acceptation des dispositifs de gestion et des outils TIC engage les professionnels au-delà de l'articulation de leurs pratiques : il s'agit aussi d'accepter l'interdépendance et une forme de contrôle (2005, p. 100). La peur du contrôle (par l'État ou par les caisses d'assurances sociales) a été constitutive de la médecine libérale à la française à la fin des années 1920. Le développement des outils relevant des TIC (systèmes d'information, DMP, Web Médecin pour l'historique des remboursements) lui donne une nouvelle forme. La rencontre des réseaux de santé et des TIC suppose la confiance de tous les utilisateurs dans les nouveaux outils TIC et la levée des peurs sur leurs usages possibles pour contrôler et limiter les soins (les rationner au lieu de les rationaliser). L'échec du carnet de santé en 1996 a montré que le succès de tout outil d'intermédiation, DMP ou autre, ne peut se construire qu'avec les médecins et les autres partenaires. La confiance dans les nouveaux dispositifs techniques devra se construire progressivement à trois niveaux: celui de la fiabilité de l'outil, des usages qui en seront faits et de sa valeur ajoutée.

\subsection{L'évaluation de la coordination comme dynamique de décloisonnement}

Longtemps peu portée au partage des données, la culture française était aussi peu favorable à l'évaluation, souvent assimilée à un contrôle vexatoire, la France n'ayant guère, à la différence des pays anglo-saxons, une culture du contrat, de l'objectif et du contrôle des résultats (D'lribarne, 1989). En s'inspirant des entreprises privées, sous l'impulsion de l'État, les choses sont en train de changer dans le secteur public. La notion d'évaluation est centrale dans les lois de 2002 et 2004. Les réseaux de santé sont désormais financés sous forme de contrats qui définissent des objectifs à atteindre. Pour M.-O. Frattini et J.-C. Mino: «jamais des services de soins n'ont été autant sous contrôle des tutelles » (2006, p. 480). La question de l'évaluation pose aussi celle de l'obsession de l'efficience, trop souvent considérée sous l'angle quasi uniquement quantitatif, comme l'a montré H. Minztberg (2001, p. 479). 


\section{Christian BOURRET}

L'évaluation est centrale dans la rencontre des réseaux de santé et des TIC. Toute évaluation suppose l'obtention rapide de données fiables pour nourrir des indicateurs pertinents. Les premières évaluations externes de réseaux de soins, évaluations contrôles et parfois sanctions, ont laissé des traces mais, désormais, l'évaluation, intégrée dans une démarche qualité, est devenue une évaluation apprenante, alliant approches internes et externes, éléments quantitatifs (nombre de patients inclus, de professionnels impliqués, d'actes réalisés, etc.) et qualitatifs (enquêtes de satisfaction).

Notre implication dans l'évaluation des réseaux de santé nous a permis de mieux appréhender les changements intervenus. Associant tous les acteurs, l'évaluation est désormais conçue comme une aide au développement du réseau et une estimation de la dynamique de décloisonnement impulsée par le réseau.

L'évaluation de ces nouvelles formes organisationnelles est avant tout celle de leur valeur ajoutée collective et donc de leur coordination. Leur évaluation met les réseaux de santé à l'épreuve d'«un arbitrage efficacité-équité » (Gadreau, 2001) entre le service rendu et l'accès aux soins. Elle est largement celle de leur système d'information (qualité des données, traçabilité des parcours de soins, etc.) et des usages des TIC. Elle va désormais s'articuler à une évaluation des pratiques personnelles (EPP) qui, avec celle de l'accréditation-évaluation des structures de santé (hôpitaux, cliniques, etc.), est une des missions principales de la nouvelle Haute Autorité de Santé (HAS) en France.

\section{Quel avenir pour les réseaux de santé ?}

\subsection{De nouvelles pratiques coopératives territorialisées impliquant les patients}

R. Bercot et F. De Coninck (2006) ont montré que, malgré les obstacles, les réseaux de santé contribuent à l'émergence de nouvelles pratiques: d'une « nouvelle médecine », plus proche d'un patient rendu plus autonome.

L'ancrage territorial est essentiel dans le développement des réseaux de santé. Selon R. Bercot (2006), en réorganisant les modalités d'intervention entre les professionnels du soin et ceux du social, le réseau de santé redessine les frontières du territoire entre la ville et l'hôpital. Pour G. Poutout, ils correspondent à une certaine forme d'« extraterritorialité » : le réseau qui « relie autour d'une personne malade l'ensemble des ressources nécessaires », n'est « ni en ville, ni à l'hôpital, ni médical, ni social, mais est tout cela à la fois » (Poutout, 2005, p. 53). 
Les réseaux de santé ou la rencontre de la santé et des TIC pour décloisonner le système de santé français

Les réseaux de santé créent de nouveaux territoires de pratiques de santé, à la fois de proximité physique de manière classique, mais aussi virtuels par les usages de l'information, notamment à travers de nouveaux outils TIC pour nourrir leurs systèmes d'information y compris par des collectes de données au chevet du patient. Un des enjeux actuels est leur articulation avec les hôpitaux de proximité et leur intégration dans les SROS 3 (Schémas Régionaux d'Organisation Sanitaire de la $3^{\mathrm{e}}$ génération). Ils peuvent contribuer à revaloriser le rôle de l'hôpital de proximité. Selon B. El Ghozi, l'implication des patients et la territorialisation des pratiques de santé sont indispensables pour éviter le risque majeur pour les réseaux de santé : «l'enfermement dans un circuit fermé de professionnels » (El Ghozi, 2005, p. 20).

\subsection{Un bilan contrasté}

Si le besoin d'organisations d'interface et d'intermédiation est incontournable (la « reliance » chère à $\mathrm{E}$. Morin et à $\mathrm{R}$. Sainsaulieu), l'avenir des réseaux de santé n'en est pas pour autant assuré.

Le rapport controversé de l'inspection générale des affaires sociales (IGAS) de mai 2006 est très critique, considérant les réseaux de santé comme «plus que décevants ", notamment en termes de services rendus aux patients et de lisibilité. Ce rapport, mal vécu par la plupart des acteurs des réseaux de santé, a justifié une diminution de leur financement, probablement au profit du projet de DMP. II admet néanmoins que les réseaux de santé « restent potentiellement intéressants». Ce rapport a encore accentué le contrôle des tutelles, en particulier par l'évaluation (circulaire de mars 2007). Pour M.-O. Frattini et J.-C. Mino, dont nous partageons l'approche, «autant qu'un service pour les professionnels et les usagers", les réseaux de santé constituent un " formidable moyen de recherche et développement, entre innovation de terrain et changement planifié, au service de l'intérêt général » (Frattini et Mino, 2006, p. 481).

En fait, comme l'a formulé $P$. Bonafini (2006), le développement des réseaux de santé relève d'une «injonction paradoxale ». Ils ont certes fait l'objet d'une reconnaissance institutionnelle dans la loi de mars 2002, mais, en même temps, trop de problèmes n'ayant pas été réglés, les réseaux de santé constituent souvent des « bricolages organisationnels » (Schweyer, 2005, p. 103).

À ce jour, au nombre de 1000 à 2000, ils regroupent au maximum 100 à 150000 patients sur tout le territoire. L'implication plus forte des patients, accélérée par les nouveaux usages des TIC, va de pair avec l'affirmation du rôle des collectivités territoriales. Lors de la rencontre des réseaux du Val de Marne (mars 2007), pour C. Saout, président d'AIDES (Association de lutte contre le 


\section{Christian BOURRET}

SIDA) et de la Conférence nationale de santé, les réseaux de santé doivent prouver leur utilité sanitaire et sociale. Ils pourraient aussi acquérir une légitimité supplémentaire en devenant des espaces de protection des droits des patients, notamment pour l'utilisation des données personnelles.

Les réseaux de santé sont à la croisée des chemins. Vont-ils devenir des structures pérennes, un nouveau mode de gestion du soin et de la santé ou, au contraire, n'auront-ils constitué qu'une étape, une modalité ponctuelle de mise en place de pratiques coopératives?

II est difficile d'échanger des données avec des personnes qu'on ne connaît pas. Les réseaux de santé peuvent constituer des espaces de construction de la confiance, de développement de nouvelles pratiques coopératives, autour de nouveaux usages des $\mathrm{TIC}$ entre des partenaires apprenant à se connaître et à travailler ensemble.

\subsection{Un indispensable maillage territorial et la coopération avec les collectivités locales}

La multiplication des réseaux pose question. Le patient ne risque-t-il pas d'être écartelé entre différents réseaux? Censés lutter contre les cloisonnements, les réseaux de santé ne risquent-ils pas d'en créer de nouveaux? Des initiatives fédératrices comme les réseaux cancer (plan national), gérontologiques, diabète, etc., sont importantes, comme les maillages régionaux de réseaux (diabète, cancer, obésité, bronchiolite, santé mentale, etc.).

La question du maillage régional des réseaux de santé et de l'intégration de leur production collective de données dans des systèmes d'information régionaux (Fieschi, 2003) est une manière d'aborder la question de leur taille critique. Certains réseaux se sont regroupés en « maisons de réseaux » comme à Saint-Denis ou à Alès (Reseda). Ces regroupements illustrent la construction très progressive des réseaux de santé : de 1985 pour le premier réseau précarité dans la cité des FrancMoisins à 2003 pour la maison des réseaux de Saint-Denis, de 1993 à 2004 à Alès.

Mais, avant de travailler ensemble, il faut apprendre à se connaître, au sein d'un même réseau et entre réseaux. Les initiatives se sont multipliées en 2007 et 2008, notamment en Île-de-France : journées des réseaux du Val de Marne, de Seine-Saint-Denis, de Seine-et-Marne ou des Hauts-de-Seine.

Les réseaux doivent avoir une meilleure lisibilité avec notamment la création d'un portail commun et d'un guichet unique, probablement au niveau régional. Un portail commun peut aussi favoriser une certaine mutualisation de moyens. Les TIC sont bien au centre de l'évolution des réseaux de santé. Elles sont 
Les réseaux de santé ou la rencontre de la santé et des TIC pour décloisonner le système de santé français

aussi essentielles pour la formation, déterminante pour une organisation apprenante, l'accompagnement des apprentissages organisationnels et la capitalisation des expériences.

Les réseaux de santé ne sont pas les seules organisations d'interface à avoir vu le jour. II existe aussi en France des structures d'hospitalisation à domicile (HAD), véritables « réseaux de réseaux », des CLIC (Centres Locaux d'Information et de Coordination), plus particulièrement destinés aux personnes âgées, des permanences de soins, des maisons de santé pluriprofessions, que les pouvoirs publics semblent vouloir privilégier.

Se pose la question de l'articulation des réseaux de santé entre eux et avec toutes les autres organisations de santé, articulation à réaliser au niveau régional, la région étant désormais considérée comme le « cadre territorial d'une régulation renouvelée » (Polton dir., 2000, p. 167). Contrairement à la plupart des autres pays européens, la régionalisation de la gestion de la santé est relativement récente en France : les URML (Unions Régionales de Médecins Libéraux) datent de 1993, les ARH (Agences Régionales de l'Hospitalisation) et les URCAM (Unions Régionales des Caisses d'Assurance Maladie), de 1996. Comme l'importance accordée aux systèmes d'information, la poursuite de la régionalisation de l'action sanitaire constitue un des axes majeurs des réformes initiées en 2004 en France.

\section{Conclusion}

Les réseaux de santé ont pour objectif essentiel de décloisonner le système de santé en constituant de nouveaux espaces de coopération entre la médecine de ville et l'hôpital, favorisant la recomposition des positionnements et des rapports de force entre acteurs traditionnels et nouveaux arrivants (patients, collectivités territoriales). Le développement de nouveaux outils relevant des TIC leur ouvre de nouvelles perspectives pour assurer la traçabilité des soins et construire de nouvelles pratiques impliquant les patients.

Le besoin d'organisations d'intermédiation pour reconstruire du lien social, avec notamment toute l'importance du milieu associatif (Sainsaulieu, 2001) pour améliorer la cohésion sociale et repenser l'État providence (Rosanvallon, 1998), a été maintes fois souligné. Pour G. Poutout, il s'agit de «créer du lien pour donner du sens » (2005, p. 33). Les réseaux de santé peuvent être considérés comme des organisations « hologrammatiques » au sens d'E. Morin et J.-L. Le Moigne (2003). Ils sont représentatifs des grands enjeux actuels : d'amélioration de la santé, de cohésion sociale et d'aménagement du territoire, mais, aussi, à travers l'utilisation via Internet des données de santé, de celui de l'avenir de 
toute notre société : société de liberté ou société du contrôle? Cette ambivalence est au coeur des réseaux de santé.

Ils constituent des espaces de négociation avec l'enjeu majeur du développement de la confiance mais ils doivent avant tout être au service du patient: "Un réseau de santé ne produit pas et ne gère pas des flux de données médicales ou sociales, il résout des problèmes médico-sociaux " (CNR, 2005, p. 73).

\section{Références bibliographiques}

AKRICH M., M. CALLON et B. LATOUR, 2006, Sociologie de la traduction. Textes fondateurs, Paris, Mines de Paris.

BERCOT R., 2006, "La coopération au sein d'un réseau de santé. Négociations, territoires et dynamiques professionnelles ", Négociations, 1, pp.35-49.

BERCOT R. et F. DE CONINCK, 2006, Les réseaux de santé, une nouvelle médecine ?, Paris, L'Harmattan, coll. Logiques sociales.

BERNOUX P., 2002, "Sociologie des organisations. Les nouvelles approches ", dans P. CABIN (coord.), Les organisations. Etat des savoirs, Auxerre, Editions Sciences Humaines. pp. 39-50.

BONAFINI P., 2006, Le développement des réseaux de santé : une injonction paradoxale?, dans Actes du colloque "La métamorphose des organisations ", Université Nancy 2 GREFIGE-CEREMO, 23-24 novembre 2006 $<$ http://www.univ-

nancy2.fr/COLLOQUES/METAMORPHOSE_06/fichiers_PDF/BONAFINI.pdf>, dernière consultation le 15 juin 2008.

BOURRET C., 2004, "Réseaux de santé et nouveaux métiers de l'information », Documentaliste - Sciences de l'information, vol. 41, $n^{\circ} 3$, pp.174181.

BOURRET C., 2002, "Les réseaux de santé : un champ de recherches prometteur pour les Sciences de l'Information et de la Communication ", dans Actes du $13^{e}$ Congrès national des sciences de l'information et de la communication, 7-9 octobre 2002, Marseille, pp.345-352.

BOUTINET J.-P., 2006, Psychologie des conduites à projet, Paris, PUF. 
Les réseaux de santé ou la rencontre de la santé et des TIC pour décloisonner le système de santé français

CABÉ M.-H. (coord.), 2005, «La santé en réseaux. Quelles innovations ?», Sociologies Pratiques, PUF, $\mathrm{n}{ }^{\circ 11 .}$

CABIN P. (coord.), 2002, Les organisations. Etat des savoirs, Auxerre, Editions Sciences Humaines.

CALLON M., 2006, "Sociologie de l'acteur réseau », dans M. AKRICH, M. CALLON et B. LATOUR, Sociologie de la traduction. Textes fondateurs, Paris, Mines de Paris, pp.267-276.

CARRÉ D. et J.-G. LACROIX (dir.), 2001, La santé et les autoroutes de l'information. La greffe informatique, Paris, L'Harmattan.

CASTELLS M., 1998, La société en réseaux, Paris, Fayard.

COMMITTEE ON GOVERNMENT REFORM, 2005, The Last Frontier: Bringing the Revolution to Health Care, Washington, http://frwebgate.access.gpo.gov/cgi-

bin/getdoc.cgi?dbname $=109$ house hearings\&docid=f:24713.wais, dernière consultation le 15 juin 2008.

COORDINATION NATIONALE DES RESEAUX DE SANTE (CNR), 2005, Réseaux de Santé : la qualité en pratique, Paris.

COUR DES COMPTES, «Le partage des données entre les systèmes d'information en santé », dans rapport sur la Sécurité Sociale, Paris, 2007, <http://www.ccomptes.fr/CC/Sommaire-18.html>, dernière consultation le 15 juin 2008, pp. 309-327.

CROZIER M. et E. FRIEDBERG, 1977, L'acteur et le système. Les contraintes de l'action collective, rééd., 2001, Paris, Editions du Seuil.

DANIEL C., B. DELPAL et C. LANNELONGUE, 2006, Contrôle et évaluation du fonds d'aide à la qualité des soins de ville (FAQSV) et de la dotation de développement des réseaux (DDR), rapport Inspection générale des affaires sociales (IGAS), Paris, La Documentation française.

EL GHOZI B., 2005, « Réseau de santé et action professionnelle », dans M.-H. CABÉ (coord.), «La santé en réseaux. Quelles innovations? », Sociologies Pratiques, PUF, n¹1, pp. 17-20.

ELLUL J., 1990, La technique ou l'enjeu du siècle, rééd., 2001, Paris, Economica.

FEENBERG A., 2004, (Re)penser la technique. Vers une technologie démocratique, Paris, La Découverte - MAUSS. 
FIESCHI M., 2003, «Les données du patient partagées: la culture du partage et de la qualité des informations pour améliorer la qualité des soins ", Rapport remis au ministre de la santé, Paris.

FRATTINI M.-O. et J.-C. MINO, 2006, Les réseaux, un outil de recherche et développement au service de l'évolution du système de santé, Santé publique, vol. $18, n^{\circ} 3$, pp. 475-481.

FRIEDBERG E., 1993, Le pouvoir et la règle, Paris, Editions du Seuil.

FUCHS V. R., 1998, Who Shall Live? Health, Economics, and Social Choice, London, World Scientific.

GADREAU M., 2001, "Les réseaux dans le système de santé et l'arbitrage efficacité-équité ", Economie Appliquée, t. LIV, n² 2, pp. 91-128.

GIDDENS A., 1987, La constitution de la société, rééd., 2005, Paris, PUF, coll. Quadrige.

GIDDENS A., 1994, Les conséquences de la modernité, rééd., 2006, Paris, L'Harmattan.

GRIMSON J., W. GRIMSON et W. HASSELBRING, 2000, « The SI challenge in Health Care ", Communications of the ACM, vol. 43, n ${ }^{\circ} 6$, pp.49-55.

GROSJEAN M., J. HENRY, A. BARCET et J. BONAMY, 2004, « La négociation constitutive et instituante. Les co-configurations du service en réseaux de soins ", Négociations, 2, pp.75-90.

GROSJEAN S. et L. BONNEVILLE, 2007, "Logiques d'implantation des TIC dans le secteur de la santé ", Revue française de gestion, n¹72, pp.145-157.

HABER S., 2006, "Confiance et lien interpersonnel de Husserl à Luhmann ", dans A. OGIEN et L. QUERE (dir.), Les moments de la confiance. Connaissance, affects et engagements, Paris, Economica, pp. 43-61.

HAUTE AUTORITE DE SANTE, 2006, Une démarche d'amélioration de la Qualité. Les réseaux de santé, Paris.

IRIBARNE P. D', 1989, La logique de l'honneur. Gestion des entreprises et traditions nationales, rééd., 2003, Paris, Editions du Seuil.

LE BOTERF G., 2004, Travailler en réseau. Partager et capitaliser les pratiques professionnelles, Paris, Editions d'Organisation.

MARCH J.G., 1991, Décision et organisation, Paris, Dunod.

MAYERE A. et B. VACHER, 2005, "Le slack, la litote et le sacré ", Revue française de Gestion, hors série, Dialogues avec James March, pp.63-86. 
Les réseaux de santé ou la rencontre de la santé et des TIC pour décloisonner le système de santé français

MIEGE B., 2004, L'information-communication objet de connaissance, Bruxelles, De Boeck.

MINTZBERG H., 2001, Le Management. Voyage au centre des organisations. Paris, Editions d'Organisation.

MORIN E. et J.-L. LE MOIGNE, 2003, L'intelligence de la complexité, Paris, L'Harmattan.

MUCCHIELLI A. et C. NOY, 2005, Etude des communications: Approches constructivistes, Paris, Armand Colin.

MUSSO P., 2003, Critique des réseaux, Paris, PUF.

NHS EXECUTIVE, 1998, Information for Health. An Information Strategy for the Modern NHS 1998 - 2005, London.

NOOTEBOOM B., 2006, "Apprendre à faire confiance ", dans A. OGIEN et L. QUERE (dir.), Les moments de la confiance. Connaissance, affects et engagements, Paris, Economica, pp. 63-85.

OGIEN A. et L. QUERE (dir.), 2006, Les moments de la confiance. Connaissance, affects et engagements, Paris, Economica.

PEAUCELLE J.-L., 2007, «La malléabilité organisationnelle des TIC », Revue française de gestion, $n^{\circ} 172$, pp.105-116.

POLTON D., (dir.), 2000, Quel système de santé à l'horizon 2020 ?, Paris, La Documentation française.

POUTOUT G., 2005, "Réseaux de santé : créer du lien pour donner du sens », dans M.-H. CABÉ (coord.), "La santé en réseaux. Quelles innovations? ", Sociologies Pratiques, PUF, n¹1, pp.33-59.

ROMANOW R. J., 2002, Guidé par nos valeurs. L'avenir des soins de santé au Canada, Commission sur l'avenir des soins de santé au Canada, Montréal, Editions du gouvernement du Canada.

ROSANVALLON P., 1998, La nouvelle question sociale. Repenser l'Etat providence, Paris, Editions du Seuil.

SAINSAULIEU R., 2001, Des sociétés en mouvement. La ressource des institutions intermédiaires, Paris, Desclée de Brouwer.

SCHWEYER F.-X., 2005, «Le travail en réseau : un consensus ambigu et un manque d'outils », dans M.-H. CABE (coord.), "La santé en réseaux. Quelles innovations? ", Sociologies Pratiques, PUF, n¹1 
SCHWEYER F.-X., 2004, "Les territoires de santé et la médecine libérale. Les enjeux d'une convergence ", Lien social et Politiques - RIAC, 52, pp.35-46.

SERIEYX H. et H. AZOULAY, groupe CFC, 1996, Face à la complexité. Mettez du réseau dans vos pyramides. Penser, organiser, vivre la structure en réseau, Paris, Village Mondial.

SFEZ L. (dir.), 2001, L'utopie de la santé parfaite, Paris, PUF.

\section{Sites Internet consultés}

Annuaire Sécu : www.annuaire-secu.com

Coordination Nationale des Réseaux : www.cnr.asso.fr

Haute Autorité de Santé : www.has-sante.fr

Ministère de la Santé : www.sante.gouv.fr

\section{Pour citer cet article}

Christian Bourret, «Les réseaux de santé ou la rencontre de la santé et des TIC pour décloisonner le système de santé français», Revue tic\&société [En ligne], Tic et santé, Numéros, mis à jour le : 14/10/2008, URL : http://revues.mshparisnord.org/lodel/ticsociete/index.php?id=396 